\title{
Management Optimization on Used Express Packages-from the Perspective of Energy and Emission Reduction
}

\author{
$\mathrm{Xu} \mathrm{BAO}^{1, \mathrm{a},{ }^{*}}$ \\ ${ }^{1}$ Department of Law and Political Science, North China Electric Power University, Baoding, China, \\ 071000 \\ 2wen163ke@163.com \\ "Corresponding author
}

Keywords: Used express packages, Management optimization, Energy and emission reduction.

\begin{abstract}
With rapid expansion of the scale of China's online shopping and fast growth of the express industry comes out concurrently a problem how to handle those used express packages. From the perspective of energy and emission reduction, the management on those used express packages can be optimized by co-operations among the government, the express businesses and the public. The government should establish the relevant law and regulations and encourage the express businesses and the public to take part in the recycling of used express packages. The express businesses should establish the system for recycling used express packages and try to adopt the new recyclable packages. Encouraged by the government, the public will actively participate, oversee the implementation and give feedback information. Ultimately a trinity mode of the government, the businesses and the public to handle the used express packages might be formed under the guidance of the government. The mode can promote resources recycling and energy and emission reduction.
\end{abstract}

\section{Introduction}

Online shopping has become a daily consumption pattern of us, which contributes to the development of the express businesses. National Postal Official statistics show that the national scale express service businesses volume totaled 9.19 billion in 2013, an increase of $61.6 \%$; businesses revenue totaled 144.17 billion yuan, an increase of $36.6 \%$. So we can see the rapid development of the express industry, but under the pleasant situations there are also problems related to energy and emission reduction in handling the used express packages (express packages refer to the additional outside packages to make the expresses convenient, strong and safe for delivering, such as the outside cartons, foams, plastic bags, wooden boxes etc.). We can see, in our life, express packages become the daily rubbishes. Nowadays, in advocating energy and emission reduction, how can we ignore the impact of these packages?

\section{Situation of handling the used express packages in our country}

\section{Simple method of handling the used express packages, resulting in resources waste and environmental pollution}

The statistic data from our surveys show that $65 \%$ of used express packages were turned into trash after using. These packages were sent to be incinerated or buried. However, we found that this approach did not reach the standard of the treatment norms. Thus, the simple approach will inevitably lead to resources waste and environmental pollution. For example, in order not to damage the goods, express companies are increasingly strengthening those packages, using plastic bags, carton packages. When these packages are abandoned, the plastic exacerbates environmental pollution (air and soil pollution) and the carton leads to deforestation and secondary pollution. In addition, sellotape (used on express packages and containing formaldehyde in it) is the PVC material which is nondegradable without 100 years. So the impact on the environment is obvious.

We found that only part of the paper packaging was recycled from the used express packages, while plastic packaging was difficult to recycle. In terms of recycling paper packaging, there are certain benefits 
- one ton of waste paper can be recycled paper $850 \mathrm{~kg}$, also save wood $300 \mathrm{~kg}$ and produce less pollution (reducing $74 \%$ of pollution than the same amount of new paper). But recovery rate for waste paper in our country is less than $40 \%$, lower for their utilization. What is worse, there are many problems in the recycling, such as a lack of recovery standards, low sorting rate, high logistics and warehousing costs, no sound recycling system and large part of waste paper depending on import and so on. Therefore, the efficiency of recycling the used express packages as waste paper is really poor. As for the used plastic express packages, there is no relevant recycling habit and they can only be incinerated or buried, so the harm of this handling way is self-evident.

\section{No uniform sectoral standards and production standards of express packages (such as material specifications, size specifications, and sorting norms)}

It is reported that many domestic express companies have their own special packaging manufacturer. There are several standards of the size of packages. The majority of businesses still use their original packaging. Now, the large-scale online retailers have their own packages. Some sellers on Taobao (Taobao was founded by Alibaba. It's a large network of retail shopping in the Asia-Pacific region. Its business contains C2C and B2C) also have their own designed packages. Some small sellers and scattered customers use used cardboard for packaging. Few sellers use the box provided by express companies. Such status has resulted in the uneven size and quality of express packages. Also, it causes that parcels can not make full use of the container space, resulting in wasting for storage space and increasing transportation costs. In the express companies, parcels are sorted by hands and the quality of the sorting personnel there is also different. We can see that there is the lack of necessary management for sorting process. Therefore, the violence in packages split has occurred sometimes, causing damage to the express packages and consumers' dissatisfaction.

\section{Lack of public awareness and participation}

For one thing, the data of our surveys shows that the public lack awareness of environmental protection. The public have not realized that the used express packages had a bad influence on the environment. To our surprise, most people think the express packages are not strong enough to protect the express items. This is because in the delivery process of express items, the items will go through several sorting processes, which needs a higher level of protection from express packages. However, now materials of express packages are mostly inferior, leading to a serious shortage of these packages - the express items are damaged. It is because of this reason that online retailers are often used to adding reinforcement or the secondary packages to reduce damage to express packages during the transport, such as increasing the sellotape on the packages and filling inside. Packaging excess increases in the workload and cost of sale, and also causes a lot of express packages waste.

For another, the surveys show that the people tend to discard the express packages. There are two main reasons. One is because of the social environment - there is no the appropriate recycling system and no social atmosphere of recycling the used express packages. The other is because the public's own private interests. They think the used express packages take up too much room or maybe collecting these packages makes them ashamed when facing others. So many people tend to discard these packages rather than recycle them. Such worrying realities will inevitably pollute the environment and waste resources, like wood, water and so forth.

\section{Management optimization for the used express packages}

The current situation of handling the used express packages reflects the fact that the government, the express businesses and the public have not yet reached a uniform consensus on the method of handling these packages. There is the lack of cooperation for the three parts. What we can see is that management mode for handling packages is unreasonable. Therefore, actions should be taken to manage and optimize the current handling mode by the government, the express businesses and the public.

\section{Establishing and improving recycling system for the used express packages}

To create this system, guidance and regulations from the government are mainly relied on, and ultimately, the express businesses (mainly packaging companies and express companies) implement it. 
Overall, the government needs to improve the relevant laws and regulations, establish classification standards for the waste, improve management and simplify departments for waste management so as to change the current status of the multiple waste management. Specifically, take incentive and punitive policies which are based on the actual market situation. For the companies which actively take their social responsibility, the government should give them some material rewards or spiritual rewards. So for one thing, it's convenient for the government to regulate the express businesses, for another, it contributes to encouraging more companies to take their social responsibility. As for those companies which have the bad performance, the government should take effective punitive measures to control environmental pollution caused by them. What's more, the government should ask relevant departments to oversee and rectify them. At the same time, professionals for express packages should be trained to provide human resources to keep packaging innovation and promote the development of the express businesses. Also, it will promote cycling use for express packages (cycling use - the used express package can be returned after the completion of once distribution and reused for another distribution). The government should devolve power to the market (packaging companies and express companies) to handle the used express packages and the government is only responsible for supervision. In this way, it will not only reduce the financial burden, but also make full use of express packages, saving resources and energy as well as reducing pollution.

\section{Establishing uniform standards for the express businesses}

Firstly, the government should oversee the express businesses to develop uniform standards and strictly limit the materials used to manufacture express packages. Monitoring and regulating the entire process of production, use and disposal for the express packages is needed. In consequence, it will help the express businesses to minimize the resources consumption and the pollution in the entire process.

Secondly, the express businesses optimize management and strengthen cooperation with each other. Packaging companies design the new packages for express companies. These packages are created by uniform standards. Their sizes and shape are different, but they can be easily assembled and discharged. The materials of the express packages are solid cardboard or plastic which is durable, environmental protection, easily degradable. These packages with recyclable filling are easy to transport and help save space. Once the uniform standards have established, express companies need to recruit packaging professionals and organize express for training. The training contains many aspects, such as how to assemble and discharge the packages and mastering the methods of recycling the used packages to reuse. It aims to improve the qualities of express and make assembly, sorting and other operations standardization. The cycled packages will be given back to the express companies. If the packages are worn, express companies will return these worn packages to packaging companies to manufacture new packages. Express companies occasionally give the feedback to packaging companies according to market demand to promote the improvement of packaging. However, there is the fact that it is difficult for express to recycle the used packages or take measures to encourage the public to give back to express because of some certain reasons in our life. Therefore, express companies can work with the third-party companies. Through the third-party, express companies can indirectly recycle packages and grasp the information from the market. For example, in a university, YTO Express works with China Telecom Hall in the campus. The recipients go to China Telecom Business Hall to take their items and YTO Express can entrust the Hall to recycle packages.

\section{Raising awareness and participation of the public}

For one thing, with the enhancement of the influence on our daily from the Internet, television, newspapers and other news media, the government should take advantage of the power from the media to enhance public awareness of environmental protection. The method, such as Public Service Ads, television programmers or other ways via the net, can vary from difference forms. Nevertheless, they all will spread the knowledge of cycling and reusing the used express packages and help the public to establish the Eco-consumption concept.

For another, the companies can take incentive measures to encourage public to participate in the new handling mode. For example, the express companies cooperate with the large-scale online retailers to establish the used express packages incentives recycling system. The retailers record the information of 
the customers' performance. When the customers take part in the recycling actively, the retailers give incentives to them, like coupons.

In addition, the public should also renew their own ideas. They ought to grasp the policies and actively participate in the used express packages recycling. As for the problems generated during using the new recyclable packages, the public should actively give feedback to the companies or the government to ensure the realization of their legitimate interests.

\section{Conclusion}

The rapid development of the China's express businesses needs the management optimization for handling the used express packages, forming the government to guide, the companies (mainly express companies and packaging companies) to implement and the public to actively participate - the trinity mode to handle the used express packages. In this handling mode, the new recyclable packages are the key to the express packages recycling system. In our country, the implementation of it has economic support and maneuverability, and economic and ecological benefits of it are far higher than the current handling mode. Thus, it allows a good improvement to the present situation of express packages. It also has a great influence on recycling the waste resources, promoting changing the waste resources to the recyclable resources.

\section{References}

[1] Cha Yan, Wang Quanquan. Research on Eco-packaging Based on Shopping Online [J]. Modern Business Trade Industry, 2012(14): 160-161.

[2] The operation of the postal sector in 2013 announced by National Post [EB/OL]. (Jan, 2014). http://www.spb.gov.cn/dtxx_15079/201401/t20140115_274540.html.

[3] Zhang Yingmin, etc. Current Situation about Municipal Solid Waste Treatment Technologies and Management Strategies [J]. Ecology and Environmental Sciences, 2011, 20(2):389-396.

[4] National Express Service Standard [EB/OL]. (Dec, 2011). http://www.spb.gov.cn/zcfg/bz/bzml/yzxybz/ 201112/t20111230_159242.html.

[5] Chen Aimin. Express Packages-a Bright Prospect [J]. Printing Industry, 2013(2):82-83.

[6] Jin Jing, Zhou Gengui. Development Strategies for the Express Businesses Based on the New Packages overall the "Green" Delivery Process [J]. Modern Marketing (Academy Version), 2011(8):171-173.

[7] Wang Cheng. Green Express Packages Suitable “Online Shopping” [J]. China Packaging, 2010 (7):52-55.

[8] Kong Jie, Wang Guirong, Wang Yanpeng. Analyzes for the Status of Recycling Waste Paper and Development Prospects [J]. Shanghai Paper, 2009(1): 56-58.

[9] An D.M., Wang Hanxin. Effective Countermeasures Analysis for the Improvement of Express Packages [J]. Value Engineering, 2013 (6): 322-323.

[10] Jin Congqi, etc. Study at the Lack of Convenience for the Way to Deliver Current Express Packages [J]. Popular Science and Technology, 2012(12): 176-167. 